\title{
Prevalencia y factor es asociados con sifilis y herpes genital en dos grupos de población femenina
}

Laura G Zamilpa-Mejía, Q FB, M en $C{ }^{(1)}$ Felipe U ribe-Salas, MC, M en $C$, ${ }^{(1)}$ Luis Juárez-Figueroa, MC, ${ }^{(1)}$ Ernesto Calderón-Jaimes, MC, MSP, ${ }^{(2)}$ Carlos J Conde-González, Q BP, M en C, Dr en C..$^{(1)}$

Zamilpa-Mejía LG, Uribe-Salas F, Juárez-Figueroa L, Calderón-Jaimes E, Conde-González CJ. Prevalencia y factores asociados con sífilis y herpes genital en dos grupos de población femenina. Salud Publica Mex 2003;45 supl 5:S617-S623.

El texto completo en inglés de este artículo está disponible en: http://www.insp.mx/salud/index.html

\section{Resumen}

Objetivo. Evaluar la frecuencia de marcado res de infección por elVHS-2 y Treponema pallidum, y su relación con algunas características de las mujeres que acuden a consulta ginecológica. Material y métodos Durante 1994 y 1995 se estudiaron 388 y 448 mujeres en sendos hospitales, el primero en Cuernavaca, Morelos, México, y el segundo en la Ciudad de México. Las participantes proporcionaron una muestra de sangre para identificar, a través de la técnica de W estern blot y las pruebas deVD RL y FTA-ABS, anticuerpos específicos contra los micro rganismos mencio nados; asimismo, contestaron un cuestionario sobre sus características socio demográficas y de comportamiento sexual. Los datos se analizaron con los paquetes estadísticos SPSS y EGRET. Resultados. Las frecuencias de anticuerpos contra el VHS-2 fueron $28.3 \%$, para las mujeres del primer hospital,y $18.1 \%$ para las del segundo. En el caso de anticuerpos contra T pallidum las frecuencias fueron de $2.3 \%$ y $1.1 \%$, respectivamente. La edad, el estado civil, la escolaridad y el número de compañeros sexuales de las mujeres estudiadas estuvieron asociados con los marcadores de infección por elVHS-2. Conclusiones Se encontraron frecuencias bajas de infección por los microrganismos estudiados en ambos grupos de mujeres. La infección por elVHS-2 estuvo asociada a periodos de exposición, comportamiento sexual y nivel socio económico de las mujeres. El texto completo en inglés de este artículo está disponible en:http://www.insp.mx/salud/ index.html

Palabras clave: infecciones sexualmente transmisibles; mujeres; México

\author{
Zamilpa-Mejía LG, Uribe-Salas F, Juárez-Figueroa L, \\ Calderón-Jaimes E, Conde-González CJ. \\ Prevalence and factors associated with syphilis \\ and genital herpes in two female population groups. \\ Salud Publica Mex 2003;45 Suppl 5:S617-S623. \\ The English version of this paper \\ is available at: http://www.insp.mx/salud/index.html
}

\begin{abstract}
A bstract
O bjective.To assess the frequency of HSV-2 and Treponema pallidum markers and their relationship with characteristics of women during gynecological outpatient visits. Material and Methods In 1994 and 1995, two populations of 388 and 448 women were studied in two hospitals; one in Cuernavaca, Morelos, México, and the other in Mexico City. Participants provided a blood specimen for detection of antibodies against HSV-2 and T pallidum using the Western blot technique and the VDRL and FTA-ABS tests. Also, participants answered a questionnaire on their sociodemographic characteristics and sexual behavior. The data were analyzed with the SPSS and EG RET statistical packages. Results The frequencies of HSV-2 antibodies were $28.3 \%$ in women from the first hospital, and $18.1 \%$ for those in the second. The frequencies of T pallidum antibodies were 2.3\% and $1.1 \%$, respectively.Age, marital status, education level, and number of sexual partners were associated with HSV-2 infection. Conclusions Low frequencies were found for infection by the microorganisms studied in both groups. HSV-2 infection was asso ciated to exposure periods, sexual behavior, and socioeconomic level. The English version of this paper is available at:http://www.insp.mx/salud/index.html
\end{abstract}

Key words: sexually transmitted infections; women; Mexico

(1) Instituto $\mathrm{N}$ acional de Salud Pública. Cuernavaca, Morelos, México.

(2) Hospital Infantil de México. México, DF, México.

Fecha de recibido: 11 de octubre de 2002 - Fecha de aprobado: 19 de mayo de 2003

Solicitud de sobretiros: Dr. Carlos J Conde González. Instituto N acional de Salud Pública. Avenida Universidad 655, colonia Santa María A huacatitlán, 62508 Cuernavaca, Morelos, México.

Correo electrónico: cjconde@ insp.mx 
L a necesidad de controlar las infecciones de transmisión sexual (ITS) ulcerativas, como la sífilis y el herpes genital, reside no sólo en las manifestaciones clínicas agudas que causan, sino también en las secuelas que las caracterizan y en el papel que juegan en la transmisión del VIH. ${ }^{1}$ Diversos estudios epidemiológicos han encontrado que aquellas infecciones que cursan con úlceras genitales pueden constituir la vía de entrada del VIH, particularmente en los casos de sífilis, chancroide y herpes simple tipo $2 .^{2-5}$ La sífilis es una infección causada por la bacteria Treponema pallidum, la cual es una espiroqueta patógena exclusiva del humano. Las manifestaciones clínicas varían dependiendo de la etapa en que se encuentre la infección. ${ }^{6}$ El herpes genital es causado habitualmente por el virus herpes simple (VHS) tipo 2 y ocasionalmente por el VHS tipo 1. Este último usualmente causa infecciones orolabiales, sin embargo, se han reportado casos de infección genital causados por este virus al ocurrir prácticas sexuales de tipo orogenital, mientras que el VHS-2 se transmite mediante contacto sexual y causa infecciones genitales. ${ }^{7}$

En México no se cuenta con suficientes estudios epidemiológicos sobre ITS ulcerativas en población femenina en edad reproductiva. Los trabajos que existen se han realizado en grupos específicos de mujeres como trabajadoras del sexo comercial (TS) o mujeres que acudieron a realizarse la prueba del VIH. Estos trabajos se han basado en la búsqueda de anticuerpos específicos contra el VHS-2 y T pallidum, a través de pruebas serológicas. ${ }^{8-12}$ A diferencia de las pruebas que identifican la presencia del agente causal de la infección en el momento del estudio, ${ }^{13}$ la utilización de pruebas serológicas responde a la necesidad de contar con marcadores que permitan evaluar el comportamiento sexual de las personas, tomando en cuenta diferentes periodos de exposición a la infección por el VHS-2 y T pallidum. ${ }^{14,15}$

En el presente trabajo se analizaron las seroprevalencias de anticuerpos específicos para las infecciones por el VHS-2 y T pallidum, así como los principales factores asociados a las seroprevalencias, en dos grupos de mujeres que acudieron a consulta ginecológica al Hospital Civil de Cuernavaca, Morelos, México (HCCM), y al Hospital Juárez, en la Ciudad de México (HJCM), durante 1994-1995.

\section{Material y métodos}

En este estudio, de diseño transversal, se incluyeron 388 mujeres que acudieron consecutivamente a consulta ginecológica al HCCM de enero a julio de 1994, y 448 que acudieron igualmente al HJCM desde agosto de 1994 hasta mayo de 1995. Previo consentimiento informado, todas las mujeres contestaron un cuestionario que con- tenía preguntas sobre sus características socioeconómicas, comportamiento sexual, uso de métodos anticonceptivos y antecedentes de ITS. A cada paciente se le tomó una muestra de sangre venosa $(10 \mathrm{ml})$, obtenida con tubos sin anticoagulante (Vacutainer, Becton-Dickinson, Estados Unidos de América). La identificación del cuestionario y las muestras de cada paciente se manejaron por medio de una clave impresa en etiquetas con código de barras.

Los sueros fueron separados por centrifugación a 2500 rpm durante 15 minutos; se distribuyeron en alícuotas de $2 \mathrm{ml}$ depositadas en crioviales, identificados por los mismos códigos de barras, y mantenidos en refrigeración por no más de cinco días y luego en congelación a $-20{ }^{\circ} \mathrm{C}$.

Para el diagnóstico serológico de sífilis se utilizó como prueba presuntiva no treponémica la de VDRL-látex (Bigaux Diagnóstica, México). Como prueba confirmatoria treponémica se utilizó la de FTA-ABS (Syphilam, Diagnostics Pasteur, Francia). Estos ensayos se realizaron de acuerdo con las instrucciones de los fabricantes. ${ }^{16,17}$

Para la detección de anticuerpos contra el VHS tipo 2 se realizó la prueba de inmunotransferencia Western blot $(\mathrm{Wb}) .{ }^{18}$ Previo a la realización de este procedimiento se infectaron células del insecto lepidóptero Spodoptera frugiperda (Sf9) con el baculovirus recombinante AcDSMgG-2. ${ }^{19}$ Este virus contiene la información genética para dar lugar a la expresión de la proteína gG2 específica del VHS-2. Así, al sonicar las células infectadas de Sf9 se liberó un extracto proteico crudo, separado posteriormente por una electroforesis en geles de poliacrilamida y transferido finalmente a filtros de nitrocelulosa. Estos se incubaron con los sueros de las participantes, y la unión de anticuerpos humanos contra la proteína gG2 se puso de manifiesto como una banda colorida específica, con PM aparente de 118 Kda, al agregar un conjugado de antiinmunoglobulina humana y fosfatasa alcalina, más el substrato de la enzima. ${ }^{18}$

La información fue capturada en una base de datos utilizando el paquete dBASEIV. Las pacientes seropositivas fueron sometidas a tratamiento convencional, por el médico del servicio en cada hospital, con penicilina ${ }^{20}$ en los casos de sífilis. Para aquellas mujeres seropositivas a herpes genital se les brindó, por miembros del equipo médico en cada servicio clínico, información sobre las características y el control de la infección.

Con la información obtenida se realizó inicialmente un análisis univariante para el establecimiento de frecuencias simples y la distribución de las variables estudiadas; otro bivariante, para conocer las asociaciones crudas en cada grupo de mujeres. También se estimaron las diferencias de prevalencias de anticuerpos por el VHS-2 y T pallidum, y la diferencia de promedios de 
edad, edad de inicio de relaciones sexuales y número de parejas sexuales, comparando ambos grupos de mujeres. Por último, se realizó un análisis multivariante por regresión logística para el cálculo ajustado de las asociaciones existentes en cada grupo de mujeres. Para tal efecto se utilizaron los programas SPSS/PC $+4.0 \mathrm{y}$ EGRET 0.25.6. ${ }^{21,22}$

\section{Resultados}

Las seroprevalencias de anticuerpos contra T pallidum y el VHS-2 en las mujeres estudiadas en el HCCM fueron $2.3 \%(9 / 388)$ y $28.3 \%(110 / 388)$, respectivamente. En las mujeres del HJCM los resultados fueron 1.1\% $(5 / 448)$ y $18.1 \%(81 / 448)$, respectivamente.
En los cuadros I y II se presentan las series detalladas de las variables estudiadas y la prevalencia de marcadores de infección por el VHS-2 para los dos grupos estudiados. Debido a que la frecuencia de anticuerpos contra T pallidum fue baja en ambos grupos de mujeres, su distribución se diluye aún más en las categorías de los diferentes indicadores estudiados (datos no mostrados del análisis bivariante). Por ello solamente se completó el análisis de los resultados para el VHS-2.

En los mismos cuadros se muestran los análisis bivariantes y multivariantes entre los marcadores de infección por el VHS-2, y las características de las mujeres. Podemos observar una tendencia significativa entre el incremento de la edad y el aumento en la probabilidad de haber resultado seropositiva a la infec-

\section{Cuadro I}

\section{Características asociadas con marcadores de infección por el VHS-2 en 388 mujeres que acudieron a consulta ginecológica al Hospital Civil de la ciudad de Cuernavaca, Morelos, México, DURANTE 1994-1995}

$\begin{array}{llllll}\text { Variable } & \text { No. } & \text { Prevalencia } & \text { RMC } & \text { IC } 95 \% & \text { RMA }\end{array}$ IC $95 \%$

\begin{tabular}{|c|c|c|c|c|c|c|}
\hline \multicolumn{7}{|c|}{ Edad (en años) } \\
\hline$<=25$ & 82 & 10.9 & 1.0 & & 1.0 & \\
\hline $26-35$ & 146 & 23.9 & 2.5 & $1.1-5.6$ & 3.2 & $1.3-7.7$ \\
\hline$>=36$ & 160 & 41.2 & 5.6 & $2.6-12.1$ & 7.0 & $2.9-17.2$ \\
\hline \multicolumn{7}{|l|}{ Estado civil } \\
\hline Casada & 308 & 25.0 & 1.0 & & & 1.0 \\
\hline Soltera & 27 & 33.3 & 1.5 & $0.6-3.4$ & 1.7 & $0.5-5.8$ \\
\hline 0 tro & 53 & 45.2 & 2.4 & $1.3-4.5$ & 2.4 & $1.2-4.9$ \\
\hline \multicolumn{7}{|l|}{ Escolaridad } \\
\hline Alta & 138 & 18,1 & 1.0 & & & 1.0 \\
\hline Media & 165 & 29.0 & 1.8 & $1.0-3.2$ & 1.7 & $0.9-3.1$ \\
\hline Baja & 85 & 43.5 & 3.4 & $1.8-6.4$ & 2.1 & $1.0-4.1$ \\
\hline \multicolumn{7}{|c|}{ Método anticonceptivo } \\
\hline N inguno & 117 & 35.9 & 1.0 & & & 1.0 \\
\hline Condón & 23 & 17.3 & 0.3 & $0.1-1.1$ & 0.6 & $0.1-2.3$ \\
\hline 0 tro & 248 & 25.8 & 0.6 & $0.3-0.9$ & 0.9 & $0.5-1.5$ \\
\hline \multicolumn{7}{|c|}{ N úmero de compañeros sexuales } \\
\hline Uno & 359 & 25.9 & 1.0 & & & 1.0 \\
\hline$>=2$ & 29 & 58.6 & 4.0 & $1.8-8.8$ & 5.9 & $2.2-16.0$ \\
\hline \multicolumn{7}{|c|}{ Escolaridad compañero sexual } \\
\hline Alta & 126 & 15.8 & 1.0 & & & \\
\hline Media & 174 & 31.0 & 2.3 & & $1.3-4.2$ & \\
\hline Baja & 68 & 47.0 & 4.7 & & $2.3-9.2$ & \\
\hline \multicolumn{7}{|c|}{ Antecedentes de ITS del compañero sexual } \\
\hline No & 319 & 25.7 & 1.0 & & & 1.0 \\
\hline No sabe & 18 & 33.3 & 1.4 & $0.5-3.9$ & 0.8 & $0.2-2.6$ \\
\hline Sí & 47 & 42.5 & 2.1 & $1.1-4.0$ & 1.9 & $0.9-3.9$ \\
\hline
\end{tabular}

RMC: razón de momios cruda

RMA: razón de momios ajustada por regresión logística

ITS: infecciones de transmisión sexual 


\section{Cuadro II}

Características asociadas con marCadores de INFECCIÓN POR EL VHS-2 en 448 MUJERES QUE ACUDIERON A consulta ginecológica al Hospital Juárez de la Ciudad de México, durante 1994-1995
Variable
No. Prevalencia
RMC
IC $95 \%$
RMA
IC $95 \%$

\begin{tabular}{|c|c|c|c|c|c|c|}
\hline \multicolumn{7}{|l|}{ Edad (en años) } \\
\hline$<=25$ & 149 & 6.0 & 1.0 & & 1.0 & \\
\hline $26-35$ & 153 & 17.7 & 3.3 & $1.5-7.3$ & 3.2 & $1.3-7.7$ \\
\hline$>=36$ & 146 & 30.8 & 6.9 & $3.2-14.8$ & 5.7 & $2.4-13.5$ \\
\hline \multicolumn{7}{|l|}{ Estado civil } \\
\hline Casada & 306 & 15.0 & 1.0 & & 1.0 & \\
\hline Soltera & 51 & 15.6 & 1.0 & $0.4-2.3$ & 1.9 & $0.6-5.3$ \\
\hline 0 tro & 89 & 30.3 & 2.4 & $1.4-4.2$ & 2.2 & $1.1-4.2$ \\
\hline \multicolumn{7}{|l|}{ Escolaridad } \\
\hline Alta & 172 & 12.7 & 1.0 & & 1.0 & \\
\hline Media & 213 & 16.9 & 1.3 & $0.7-2.4$ & 1.7 & $0.9-3.5$ \\
\hline Baja & 62 & 35.4 & 3.7 & $1.8-7.4$ & 3.3 & $1.4-7.3$ \\
\hline \multicolumn{7}{|c|}{ Método anticonceptivo } \\
\hline $\mathrm{N}$ inguno & 264 & 15.1 & 1.0 & & & 1.0 \\
\hline Condón & 12 & 8.3 & 0.5 & $0.1-4.2$ & 0.9 & $0.1-7.9$ \\
\hline 0 tro & 167 & 22.7 & 1.6 & $1.0-2.7$ & 1.4 & $0.8-2.5$ \\
\hline \multicolumn{7}{|c|}{ N úmero de compañeros sexuales } \\
\hline Uno & 328 & 13.1 & 1.0 & & 1.0 & \\
\hline$>=2$ & 120 & 31.6 & 3.0 & $1.8-5.0$ & 2.7 & $1.4-5.0$ \\
\hline \multicolumn{7}{|c|}{ Escolaridad compañero sexual } \\
\hline Alta & 182 & 15.3 & 1.0 & & & \\
\hline Media & 208 & 18.2 & 1.2 & $0.7-2.0$ & & \\
\hline Baja & 46 & 30.4 & 2.4 & $1.1-5.0$ & & \\
\hline \multicolumn{7}{|c|}{ Antecedentes de ITS del compañero sexual } \\
\hline No & 359 & 18.1 & 1.0 & & 1.0 & \\
\hline No sabe & 59 & 11.8 & 0.6 & $0.2-1.4$ & 0.5 & $0.2-1.4$ \\
\hline Sí & 13 & 38.4 & 2.8 & $0.8-8.9$ & 2.2 & $0.5-8.7$ \\
\hline
\end{tabular}

RMC: razón de momios cruda

RMA: razón de momios ajustada por regresión logística

ITS: infecciones de transmisión sexual

ción herpética en ambos grupos de mujeres. Respecto al estado civil se encontró que las mujeres divorciadas, separadas, viudas y en unión libre (incluidas en la categoría "otro"), tanto del HCCM como del HJCM, tuvieron una razón de momios de infección herpética significativamente mayor respecto de las mujeres solteras y casadas.

La escolaridad de las mujeres y del compañero sexual fue dividida en tres categorías: alta, que incluyó a personas que habían concluido el nivel escolar de bachillerato o profesional; media, que incluyó a personas con primaria completa o con estudios de secundaria, $y$ baja, que incluyó personas que no fueron a la escuela o no habían terminado la primaria. Se observó una rela- ción inversa entre el nivel de escolaridad y la probabilidad de infección herpética en ambos grupos de mujeres. Lo mismo ocurrió con el nivel de escolaridad del compañero sexual. Ese último indicador no fue incluido en el modelo final de regresión logística, para evitar efectos de colinearidad con el indicador de escolaridad de las mujeres (se encontró una correlación significativa entre escolaridad del compañero y escolaridad de la mujer y cuyos coeficientes fueron $r=0.60$ y $r=0.51$ para el HCCM y el HJCM, respectivamente).

La razón de momios de infección por el VHS-2 fue significativamente mayor entre las mujeres con dos o más compañeros sexuales, respecto de las que refirieron tener solamente uno. No se encontró relación signi- 
ficativa entre la probabilidad de infección por el VHS-2 y la utilización de métodos anticonceptivos, ni con el antecedente de infecciones de transmisión sexual.

En lo referente al uso de métodos anticonceptivos, $59.5 \%$ (264/443) de las mujeres del HJCM y $30.1 \%$ (117/388) de las mujeres del HCCM no utilizaron ninguno de ellos. En el caso específico del uso de condón, las prevalencias de su uso entre las mujeres de Cuernavaca fue de $5.9 \%$, en comparación con $2.7 \%$ de las mujeres del Distrito Federal. Esta diferencia, calculada por el método de diferencia de proporciones, mostró ser significativa con un valor de $p=0.014$. Ahora bien, la seroprevalencia del VHS-2 en las mujeres que emplearon el condón como método de planificación familiar resultó ser más baja que en aquellas que no utilizaron ningún método y con las que usaron otro en el caso de ambas ciudades; aunque esta diferencia no resultó significativa (cuadros I y II).

El cuadro III muestra que las mujeres estudiadas en el HCCM tuvieron prevalencias significativamente mayores de infección por el VHS-2 y T pallidum, respecto de aquellas estudiadas en el HJCM. También se muestra que las mujeres del HCCM tuvieron en promedio mayor edad y menor número de parejas sexuales, en relación con las mujeres del HJCM. No hubo diferencia en relación con la edad de inicio de relaciones sexuales entre ambos grupos de mujeres.

En el cuadro IV se ilustra que hubo diferencias significativas entre la escolaridad y el antecedente de ITS de los compañeros sexuales de las participantes, pues

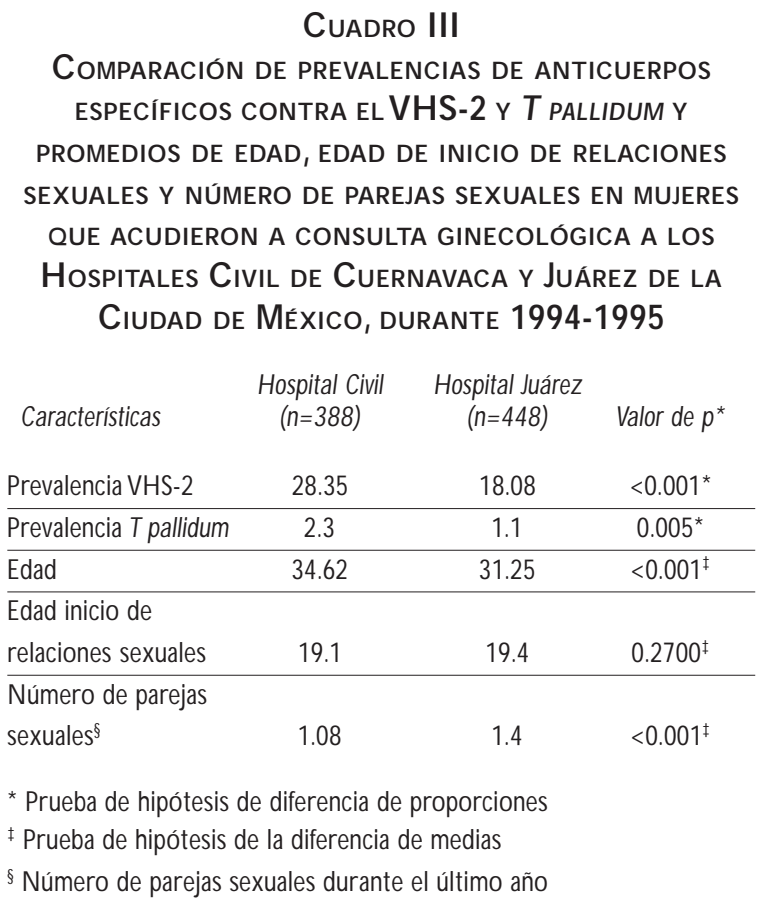

salud pública de méxico / vol.45, suplemento 5 de 2003 los hombres de Cuernavaca presentaron menor nivel educativo y un mayor historial de ITS.

\section{Discusión}

Los aportes del presente trabajo son tres. El primero muestra que las mujeres estudiadas tuvieron frecuencias bajas de marcadores de infección por T pallidum y el VHS-2. La seroprevalencia de anticuerpos específicos contra T pallidum fue de $2.3 \%$ y $1.1 \%$ para el HCCM y el HJCM, respectivamente. Estos resultados confirman que se trata de poblaciones con conducta sexual de bajo riesgo para contraer la infección por T pallidum, ya que las seroprevalencias son significativamente menores a las reportadas en TS en México, las que han variado entre $8.2 \%$ y $27.8 \%$. $^{8-11}$

Asimismo, las seroprevalencias de anticuerpos específicos contra el VHS-2 fueron $28.3 \%$ y $18.1 \%$ para las mujeres estudiadas en los HCCM y HJCM, respectivamente. Estas frecuencias difieren significativamente de las reportadas en TS de la Ciudad de México, las cuales variaron entre $60.8 \%$ y $65.1 \%$ en estudios de reciente publicación. ${ }^{15,23}$

Un segundo aporte se refiere al conocimiento de los indicadores asociados con periodos de exposición a la infección por el VHS-2 (utilizando anticuerpos específicos contra el VHS-2), entre los que destaca la edad. Una explicación a este fenómeno sería que la edad se encuentra relacionada, tanto con el número de años de actividad sexual, como con el número de parejas

\section{Cuadro IV}

Comparación de La escolaridad y el ANTECEDENTE DE INFECCIONES DE TRANSMISIÓN SEXUAL EN LOS COMPAÑEROS SEXUALES DE LAS MUJERES ESTUDIADAS EN los Hospitales Civil de Cuernavaca y JuÁrez de la Ciudad de México, durante 1994-1995

$\begin{array}{ccc}\text { Hospital Civil } & \text { Hospital Juárez } \\ \text { Características } & (n=388) & (n=448)\end{array}$ Valor de $p^{*}$

Escolaridad del

compañero sexual

\begin{tabular}{llll} 
Buena & 34.24 & 41.74 & \\
\hline Regular & 47.28 & 47.71 & 0.003 \\
\hline Mala & 18.48 & 10.55 & \\
\hline Antecedentede & & &
\end{tabular}

Antecedente de ITS

del compañero sexual

\begin{tabular}{lccc} 
No & 83.07 & 83.29 & \\
\hline No sabe & 4.69 & 13.69 & $<0.001$ \\
\hline Sí & 12.24 & 3.02 &
\end{tabular}

* Prueba de independencia de $\chi^{2}$ 
sexuales que un individuo podría tener en un momento determinado de su vida, reflejando por ello periodos de exposición al virus. ${ }^{24}$ En otras palabras, el incremento de la edad incrementa el riesgo de infección por el virus, como lo muestran los resultados del presente trabajo.

Un tercer aporte consiste en mostrar que, en nuestro medio, la identificación de anticuerpos específicos contra el VHS-2 en suero representa, en estudios epidemiológicos, un indicador de comportamiento sexual de riesgo. La identificación de anticuerpos específicos contra el VHS-2 mediante técnicas de Western blot está haciendo alusión al contacto que el individuo estudiado ha tenido con el virus en el pasado. Este hecho ha dado pie a que algunos autores utilicen dicha técnica como un marcador del estilo de vida sexual en poblaciones. ${ }^{25}$ En el presente trabajo se encontró que si bien las mujeres tuvieron en promedio bajas tasas de cambio en el número de parejas sexuales (1.085 en el HCCM y 1.4 en el HJCM), aquellas con dos o más parejas tuvieron razones de momios significativamente mayores de infección por el VHS-2 que quienes sólo reportaron una pareja sexual durante el tiempo evaluado. Ya en un estudio previo discutimos la utilidad epidemiológica de este marcador para evaluar comportamiento sexual de riesgo entre diferentes grupos poblacionales en México. ${ }^{15}$ En el presente trabajo mostramos además que aun dentro de poblaciones con frecuencias bajas de infección por el VHS-2 el marcador de esta infección puede discriminar, entre los individuos estudiados, comportamiento sexual de riesgo.

Como es bien sabido, el uso del preservativo es una variable importante para la prevención de las ITS ulcerativas; en consecuencia, este punto fue abordado indirectamente en el estudio con el fin de observar el efecto protector del condón. En primera instancia se pudo apreciar una frecuencia baja de su uso entre las participantes en ambas ciudades, y si bien la seroprevalencia del VHS-2 fue menor al comparar usuarias contra no usuarias de condón (de $17.3 \%$ vs $35.9 \%$ en el HCCM, y de $8.3 \%$ vs $15.1 \%$ en el HJCM), el hallazgo careció de significancia estadística, muy probablemente debido al escaso número de mujeres que utilizaron el condón y a la forma como se evaluó su uso.

Las mujeres del HCCM tuvieron frecuencias significativamente mayores de ambas infecciones, respecto de las estudiadas en el HJCM (cuadro III). Este fenómeno podría ser explicado por el hecho de que las mujeres estudiadas en el HCCM tuvieron en promedio mayor edad respecto de las estudiadas en el HJCM. Ya se mostró que la edad se encuentra relacionada con la frecuencia de marcadores de infección por el VHS-2. Sin embargo, el efecto fue contrario en relación con el com- portamiento sexual. Las mujeres del HJCM tuvieron en promedio mayor número de parejas sexuales durante el último año que las del HCCM. Este resultado podría deberse a que en este estudio solamente se evaluó el número de parejas sexuales en el último año (el previo a la aplicación de la encuesta). Una limitación fue no haber evaluado el número de parejas sexuales durante toda la vida, lo que podría haber mostrado el posible efecto de la edad en el comportamiento sexual de las mujeres.

No obstante, evaluamos algunas características de los compañeros de las mujeres con el fin de establecer contrastes que explicaran las diferencias en las prevalencias observadas entre ambos grupos. Observamos en el cuadro IV que los compañeros de las mujeres del HCCM tuvieron un nivel de escolaridad menor respecto de los compañeros de las del HJCM. Para utilizar este indicador probamos primero la existencia de concordancia entre la escolaridad de las mujeres y la de sus compañeros sexuales, utilizando la prueba estadística de gama (0.7882 para el HCCM, y 0.7313 para el HJCM). Las observaciones sobre la escolaridad de los compañeros sexuales de las mujeres estudiadas coinciden con los resultados que muestran que la razón de momios de infección por el VHS-2 fue significativamente mayor en las mujeres con menor escolaridad en ambos grupos estudiados (cuadros I y II). El hecho de que el nivel de escolaridad de los compañeros sexuales de las mujeres del HCCM fuera menor que el correspondiente para las del HJCM, genera la hipótesis de que una mayor prevalencia de anticuerpos contra los agentes estudiados en el primer grupo de mujeres corresponda a una mayor probabilidad de infección a partir de sus parejas sexuales. Otro argumento en este sentido fue que el antecedente de ITS en el compañero sexual de las mujeres estudiadas en el HCCM fue significativamente mayor (12.2\%) que el caso de las estudiadas en el HJCM (3.02\%).

Tomando en cuenta el diseño de investigación utilizado en este trabajo es necesario considerar sus limitaciones. En primer lugar, se trata de un estudio cuyos resultados son válidos para los grupos de mujeres estudiados. En segundo lugar, que el tipo de muestreo en ambos hospitales fue por conveniencia, por ello ambas muestras carecen de representatividad de la población femenina que acude a los nosocomios mencionados.

Los resultados en los dos grupos estudiados son de relevancia, porque si bien muestran que las mujeres tuvieron seroprevalencias de VHS-2 y sífilis bajas, en comparación con otros grupos de mayor riesgo, como las trabajadoras del sexo comercial, aun así existen las condiciones etiológicas y las conductas sexuales riesgosas para justificar la necesidad de intervenir en el nivel de los servicios de salud, con el fin de prevenir y con- 
trolar a las ITS ulcerativas en poblaciones como las aquí analizadas. ${ }^{7,26}$

\section{Referencias}

1. Brunham RC, Plummer FA.A general model of sexually transmitted disease epidemiology and its implications for control. En: Martin DH, Ed. The Medical C linics of N orth A merica: Sexually Transmitted D iseases. Filadelfia (PA):W B Saunders Company, 1990.

2.W asserheit JN . Interrelationships between HIV infection and other sexually transmitted diseases. Sex Transm D is 1992;19:61-67. 3. Hook EW, Cannon RO, N ahmias AJ, Lee FF, Campbell CH, Glasser D et al. Herpes simplex virus infection as a risk factor for human immunodeficiency virus infection in heterosexuals. I Infect D is 1992:165:251-255.

4. Boulos R, Ruff AJ, N ahmias A, Holt E, Harrison L, Magder L et al. Herpes simplex virus type 2 infection, syphilis, and hepatitis $B$ virus infection in Haitian women with human immunodeficiency virus type I and human T lymphotropic virus type I infections. J Infect D is 1992;166:418-420.

5. D ada AJ,A Aayi AO, D iamondstone L, Q uinn TC , Blattner W A, Biggar RJ. A serosurvey of Haemophilus ducreyi, syphilis and herpes simplex virus type 2 and their association with human immunodeficiency virus among female sex workers in Lagos, N igeria. Sex Transm D is 1998;25:237-242. 6. C alderón JE, C onde-G onzález C. Enfermedades de transmisión sexual. En:Arredondo GJL, Calderón JE, ed. Conceptos clínicos de infectología. 10a ed. México, D F: Méndez Editores, 1993.

7. Brugha R, Keersmaekers K, Renton A, Meheus A. Genital herpes infection:A review. Int J Epidemiol 1997;26:698-709.

8. Hyams KC, Escamilla J, Lozada R, Macareno E, Bonilla N, Papadimos TJ et al. Hepatitis $B$ infection in a non-drug abusing prostitutes population in Mexico. Scand J Infect D is 1990;22:527-531.

9.Valdespino JL, Loo E, Cruz C, G arcía ML, Magis C, Herrera C et al. Risk factors interrelated between AIDS and STD among female prostitutes, Mexico.VII International C onference on AID S; 1991 junio 16-21; Florencia, Italia.

10. Gallegos FS. Experiencia en la detección de enfermedades de transmisión sexual en un grupo de mujeres prostitutas. IV Congreso N acional de Investigación en Salud Pública; 1993 enero 25-27; Cuernavaca, Morelos, México.

11. Calderón-Jaimes E, Conde-G onzález C, Juárez-Figueroa L, U ribeZúñiga P, U ribe-Salas F, 0 lamendi-Portugal $M$ et al. Prevalencia de anticuerpos antitreponémicos en 3098 mujeres dedicadas a la prostitución en la Ciudad de México. Rev Invest Clin 1994;46:431-436.
12. Hernández-Girón C, U ribe-Salas F, Conde-G onzález C, C ruz-Valdez A, Juárez-Figueroa L, U ribe-Zúñiga $P$ et al. Seroprevalencias a diversos virus y características sociodemográficas en mujeres que buscan detectarse VIH. Rev Invest Clin 1997;49:5-13.

13. Echániz G, Conde C, Tamayo E, Cruz A, Hernández P, C alderón R. Presencia del virus herpes simple-2 (HSV-2) en mujeres que asisten a una clínica de control de cáncer cervicouterino. G inecol 0 bstet Mex 1992;60:281-285.

14. Uribe-Salas F, D el Río-C hiriboga C, Conde-G onzález C, JuárezFigueroa L, U ribe-Zúñiga P, C alderón-Jaimes E et al. Prevalence, incidence and determinants of syphilis in female comercial sex workers in Mexico City. Sex Transm D is 1996;23:120-126.

15. U ribe-Salas F, Hernández-A vila M, Juárez-Figueroa L, C ondeGonzález CJ, U ribe-Zúñiga P. Risk factors for herpes simplex virus type 2 infection among female comercial sex workers in Mexico City. Int J STD AIDS 1999;10:105-111.

16. Anónimo. Instructivo de laboratorio para el serodiagnóstico de sífilis. VD RL-látex. Marnes La Coquette: D iagnostics Pasteur, 1993.

17.A nónimo. Instructivo para el diagnóstico de anticuerpos contra T. pallidum. Syphilam. Marnes La C oquette: Diagnostics Pasteur, 1993. 18. Sánchez MD, Scott SD, W hittington W, Brown D, Reeves W C, Chatterjee $S$ et al. Evaluation of a test based on baculovirus-expressed glycoprotein $\mathrm{G}$ for detection of herpes simplex virus type-specific antibodies. I Infect D is 1991;164:1196-1199.

19. Sánchez MD, Pellet PE. Expression of HSV-1 and HSV-2 glycoprotein $G$ in insect cells by using a novel baculovirus expression vector.Virology 1991;182:229-238.

20. Centers for D isease Control and Prevention. 1998 guidelines for the treatment of sexually transmitted diseases. MMW R Morb Mortal W kly Rep 1998;47(N 0. RR-1):28-34.

21. N orusis M. SPSS/PC base manual for the IBMpc/xt/at and ps2. Chicago: SPSS, 1992.

22. EG RET. Reference manual. Seattle: Statistics and Epidemiology Research Corp, 1990.

23. C onde-G onzález CJ, Juárez-Figueroa L, U ribe-Salas F, Hernández$N$ evárez $P$, Schmid DS, C alderón $E$ et al. A nalysis of herpes simplex virus 1 and 2 infection in women with high risk sexual behavior in Mexico. Int J Epidemiol 1999;28:571-576.

24. Kots K, Forrest JD. American women's sexual behavior and exposure to the risk of sexually transmitted diseases. Fam Plann Perspect 1992;24:244-254.

25. Cowan FM, Johnson AM,A shley R, Corey L, Mindel A. Antibody to herpes simplex virus type 2 as serological marker of sexual lifestyle in populations. Br Med J 1994;309:1325-1329.

26. Elías $C$. Sexually transmitted diseases and the reproductive health of women in developing countries. N uevaYork (NY): Population Council, 1991; Programs Division W orking Paper N 0. 5. 